hep-ph/9806345

CERN-TH/98-156

DFTT 28/98

GeF-TH-6/98

\title{
ON POSITIVITY OF PARTON DISTRIBUTIONS
}

\author{
Guido Altarelli \\ Theory Division, CERN, CH-1211 Geneva 23, Switzerland; \\ Università di Roma Tre, Rome, Italy \\ Stefano Forte \\ INFN, Sezione di Torino, via P. Giuria 1, I-10125 Torino, Italy \\ Giovanni Ridolfi \\ INFN, Sezione di Genova, via Dodecaneso 33, I-16146 Genova, Italy
}

\begin{abstract}
We discuss the bounds on polarized parton distributions which follow from their definition in terms of cross section asymmetries. We spell out how the bounds obtained in the naive parton model can be derived within perturbative QCD at leading order when all quark and gluon distributions are defined in terms of suitable physical processes. We specify a convenient physical definition for the polarized and unpolarized gluon distributions in terms of Higgs production from gluon fusion. We show that these bounds are modified by subleading corrections, and we determine them up to NLO. We examine the ensuing phenomenological implications, in particular in view of the determination of the polarized gluon distribution.
\end{abstract}

Submitted to: Nuclear Physics B

CERN-TH/98-156

June 1998 


\section{Introduction}

In the naive parton model, parton distributions are interpreted as probability densities for partons to be found inside a given target [1]. The partonic viewpoint immediately implies that if the individual parton distributions are bona fide probability densities, they should all be positive. Consequently, polarized distributions, which are differences of distributions of partons with polarization parallel or antiparallel to their parent hadron, should be bounded by the corresponding unpolarized distributions, which are sums over polarizations. Because unpolarized distributions are known rather accurately, such bounds are potentially useful in the determination of the polarized distributions, and in particular of the polarized gluon distribution, which at present can only be indirectly extracted from scaling violations [2]. It is well-known that, in perturbative QCD, the naive parton model is only recovered in the asymptotic limit, when all perturbative corrections can be neglected, while at finite energy the naive parton model results, in general, receive calculable perturbative corrections. It is thus interesting to ask to which extent the naive positivity bounds for polarized densities are modified by QCD corrections, especially in the case of the gluon distribution, which is of special phenomenological interest.

Here we address this question by deriving bounds on the polarized quark and gluon distributions from positivity of cross sections. We show that the naive positivity bounds can be derived from leading order (LO) QCD, provided that the quark and gluon distributions are defined in terms of polarized and unpolarized physical processes. While it is clear that an adequate choice of defining process for quark densities is in terms of deep-inelastic structure functions, such as, e.g., $F_{2}$ and $g_{1}$, the selection of a suitable process for the gluon is subtler. In fact, in order to directly obtain the naive bound on $|\Delta g| / g$ at LO, we need a process where only gluons of a given polarization contribute at LO. Thus, one is led to consider the production of a scalar particle (such as the Higgs) in gluon-proton collisions: only the gluon in the proton contributes at LO, and, moreover, if the external gluon and the target are polarized, only one polarization state for the gluon parton is selected.

Next, we discuss how these bounds get modified by next-to-leading order (NLO) corrections, in general in a scheme-dependent way. In a generic scheme (such as the commonly used $\overline{\mathrm{MS}}$ scheme) the NLO bounds may be more or less restrictive than the LO ones, depending on the parton distribution and the region of $x$ which are considered. We then determine the full set of NLO bounds for quark and gluon partons explicitly. This requires the determination of the NLO corrections in the polarized case to the Higgs production process which defines the gluon at LO. After having established the improved NLO bounds, we use them to extract phenomenological information on polarized parton distributions in terms of the unpolarized ones, and specifically to constrain the polarized gluon distribution.

\section{Positivity of parton distributions at leading order}

In perturbative QCD, positivity bounds on parton distributions follow from their definitions in terms of cross-section asymmetries, and from positivity of physical cross sections. Let us first consider the simple case of the structure function $g_{1}\left(x, Q^{2}\right)$ for inclusive deepinelastic scattering of longitudinally polarized leptons on a longitudinally polarized target, 
which leads to bounds on polarized quark and antiquark distributions. Neglecting power corrections, $g_{1}$ is related to the asymmetry $A_{1}$ for deep-inelastic scattering of transversely polarized virtual photons on a longitudinally polarized nucleon through [3]

$$
A_{1} \equiv \frac{\sigma_{1 / 2}-\sigma_{3 / 2}}{\sigma_{1 / 2}+\sigma_{3 / 2}}=\frac{g_{1}\left(x, Q^{2}\right)}{F_{1}\left(x, Q^{2}\right)},
$$

where the subscripts denote the total angular momentum of the photon-nucleon pair along the incoming lepton's direction. This immediately implies that $g_{1}$ is bounded by its unpolarized counterpart $F_{1}$ :

$$
\left|g_{1}\left(x, Q^{2}\right)\right| \leq F_{1}\left(x, Q^{2}\right) .
$$

The structure functions $F_{1}$ and $g_{1}$ are related to parton distributions through coefficient functions according to

$$
\begin{aligned}
& F_{1}\left(x, Q^{2}\right)=\frac{1}{2} \sum_{i=1}^{n_{f}} e_{i}^{2} C_{i}^{d} \otimes\left(q_{i}+\bar{q}_{i}\right)+2 n_{f}\left\langle e^{2}\right\rangle C_{g}^{d} \otimes g \\
& g_{1}\left(x, Q^{2}\right)=\frac{1}{2} \sum_{i=1}^{n_{f}} e_{i}^{2} \Delta C_{i}^{d} \otimes\left(\Delta q_{i}+\Delta \bar{q}_{i}\right)+2 n_{f}\left\langle e^{2}\right\rangle \Delta C_{g}^{d} \otimes \Delta g
\end{aligned}
$$

where $\otimes$ denotes the usual convolution with respect to $x, q_{i}$ and $\bar{q}_{i}$ are quark and antiquark distributions of flavour $i$ and with electric charge $e_{i},\left\langle e^{2}\right\rangle \equiv \frac{1}{n_{f}} \sum_{i=1}^{n_{f}} e_{i}^{2}, g$ is the gluon distribution, $\Delta q_{i}, \Delta \bar{q}_{i}$ and $\Delta g$ the corresponding polarized distributions, and $\Delta C^{d}$ and $C^{d}$ are, respectively, polarized and unpolarized coefficient functions (the index $d$ is a reminder that these are deep-inelastic coefficient functions). The coefficient functions can be written as series in $\alpha_{s}$ with $x$-dependent coefficients:

$$
C\left(x, \alpha_{s}\right)=\sum_{k=0}^{\infty}\left(\frac{\alpha_{s}}{2 \pi}\right)^{k} C^{(k)}(x)
$$

Now, at LO

$$
\begin{aligned}
& C_{i}^{d,(0)}(x)=\Delta C_{i}^{d,(0)}(x)=\delta(1-x) \\
& C_{g}^{d,(0)}(x)=\Delta C_{g}^{d,(0)}(x)=0,
\end{aligned}
$$

so that at LO eq. (2.2) immediately implies

$$
\left|\sum_{i=1}^{n_{f}} e_{i}^{2}\left[\Delta q_{i}\left(x, Q^{2}\right)+\Delta \bar{q}_{i}\left(x, Q^{2}\right)\right]\right| \leq \sum_{i=1}^{n_{f}} e_{i}^{2}\left[q_{i}\left(x, Q^{2}\right)+\bar{q}_{i}\left(x, Q^{2}\right)\right] .
$$

The bound eq. (2.7) must be satisfied as a matter of principle for any choice of target, i.e. for any combination of quark plus antiquark distributions, and thus it must be satisfied by each quark flavour separately. Furthermore, the bound must also be satisfied by the 
structure functions for charged-current scattering, and thus by each quark and antiquark distribution separately. Therefore, we conclude that at LO

$$
\left|\Delta q_{i}\left(x, Q^{2}\right)\right| \leq q_{i}\left(x, Q^{2}\right)
$$

for all flavours $i$, for all $x$, and for all $Q^{2}$ such that the LO approximation makes sense.

The constraint eq. (2.7) immediately implies that at LO it is possible to interpret the polarized and unpolarized quark distributions according to a naive parton picture. Namely, if we define distributions of partons polarized parallel or antiparallel to their parent hadron by

$$
q_{i}^{ \pm}=\frac{1}{2}\left(q_{i} \pm \Delta q_{i}\right), \quad \bar{q}_{i}^{ \pm}=\frac{1}{2}\left(\bar{q}_{i} \pm \Delta \bar{q}_{i}\right),
$$

then eq. (2.8) implies that $q_{i}^{ \pm}$and $\bar{q}_{i}^{ \pm}$are positive-(semi)definite and can thus be interpreted as probability distributions.

Next, one may ask whether a LO positivity bound can likewise be derived for the gluon distribution. In order to do so, we must select a pair of polarized and unpolarized processes which at LO define the gluon, i.e. processes whose LO coefficient functions are in an analogous way given by

$$
\begin{aligned}
& C_{i}^{h,(0)}(x)=\Delta C_{i}^{h,(0)}(x)=0 \\
& C_{g}^{h,(0)}(x)=\Delta C_{g}^{h,(0)}(x)=\delta(1-x) .
\end{aligned}
$$

Because the gluon only couples strongly, the leading nontrivial order must be at least $O\left(\alpha_{s}\right)$, unlike the quark which can be defined at $O\left(\alpha_{s}^{0}\right)$.

Processes which are typically used to determine the gluon distribution experimentally by singling out the photon-gluon fusion diagram, such as heavy quark production, are not useful to our present purpose, because even though they do have the property that at LO only the gluon coefficient functions are nonvanishing, they do not satisfy the requirement that the spin-flip coefficient function vanishes, i.e. $C_{g}=\Delta C_{g}$. Therefore, with this definition positivity of the physical cross sections does not automatically imply positivity of the parton distribution. One could also think of using deep-inelastic scattering mediated by longitudinally polarized photons, i.e. in the unpolarized case the structure function $F_{L}$, which receives a LO gluon contribution (of order $\alpha_{s}$ ) [4] (of order $\alpha_{s}$. However, there is no suitable polarized counterpart of $F_{L}$.

Nevertheless, the bound

$$
\left|\Delta g\left(x, Q^{2}\right)\right| \leq g\left(x, Q^{2}\right)
$$

is directly obtained at LO if we define the gluon distribution from inclusive Higgs production in gluon-proton scattering, i.e. from the process $g+p \rightarrow H+X$. At leading nontrivial order [i.e. $O\left(\alpha_{s}^{2}\right)$ ] this process proceeds through $g+g \rightarrow H$, induced by coupling of the gluons to a top quark loop (see fig. 1a). Even though this process is not realistic for an experimental measure of the gluon distribution, it is particularly convenient to formally define the gluon distribution and establish positivity bounds. The cross section for the LO partonic unpolarized process is given by [5]

$$
\sigma(g g \rightarrow H)=\frac{\alpha_{s}^{2}\left(m_{h}^{2}\right) G_{F} m_{h}^{2}}{288 \sqrt{2} \pi}|A|^{2} \delta\left(s-m_{h}^{2}\right),
$$



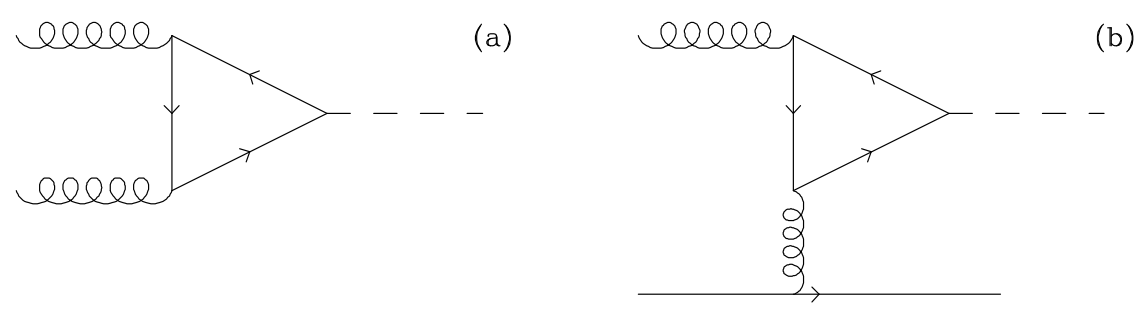

(c)

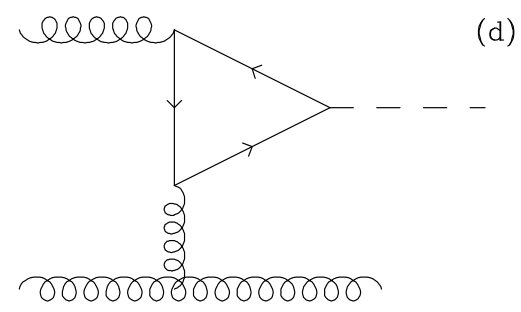

Figure 1: Diagrams for the processes (a) $g+g \rightarrow H$, (b) $g+q \rightarrow H+q$, (c, d) $g+g \rightarrow H+g$.

where $s$ is the center-of-mass energy of the $g g$ collision, and $A$ is a dimensionless function of the Higgs mass and the top quark mass: $A=A\left(m_{t}^{2} / m_{h}^{2}\right)$, such that $\lim _{r \rightarrow \infty}|A(r)|^{2}=1$. Furthermore, since the Higgs particle is scalar, only the polarized amplitude in which the two gluons have the same helicity is nonzero at LO in any frame where the two gluon momenta are in opposite directions.

We can thus define the polarized and unpolarized gluon distributions by considering the gluon-proton scattering process. It is important to note that the external gluon is to be treated as a hadron, in the sense that it must be endowed with its own nonperturbative parton densities $g_{g}, \Delta g_{g}, u_{g}, \Delta u_{g}$ and so forth. The collinear singularities associated with the initial gluon leg have then to be absorbed into a redefinition of the associated parton distributions. We are however free to choose the resulting distributions in a form which is convenient for our conceptual purposes. Our choice corresponds to that of an effective free gluon, i.e. $g_{g}(x)=\Delta g_{g}(x)=\delta(1-x), u_{g}=\Delta u_{g}=\ldots=0$. As a consequence, the gluon-proton cross section can be written as

$$
\begin{gathered}
\sigma[g p \rightarrow H+X]\left(x, m_{h}^{2}\right)=\frac{\alpha_{s}^{2}\left(m_{h}^{2}\right) G_{F}}{288 \sqrt{2} \pi}|A|^{2} \int_{x}^{1} \frac{d y}{y}\left\{C_{g}^{h}\left(\frac{x}{y}\right) g\left(y, m_{h}^{2}\right)\right. \\
\left.+C_{s}^{h}\left(\frac{x}{y}\right) \Sigma\left(y, m_{h}^{2}\right)\right\} \\
\Delta \sigma[g p \rightarrow H+X]\left(x, m_{h}^{2}\right)=\frac{\alpha_{s}^{2}\left(m_{h}^{2}\right) G_{F}}{288 \sqrt{2} \pi}|A|^{2} \int_{x}^{1} \frac{d y}{y}\left\{\Delta C_{g}^{h}\left(\frac{x}{y}\right) \Delta g\left(y, m_{h}^{2}\right)\right. \\
\left.+\Delta C_{s}^{h}\left(\frac{x}{y}\right) \Delta \Sigma\left(y, m_{h}^{2}\right)\right\},
\end{gathered}
$$


where $x=\frac{m_{h}^{2}}{s}$ and the singlet quark distribution is defined as

$$
\Sigma\left(x, Q^{2}\right)=\sum_{i=1}^{n_{f}}\left[q_{i}\left(x, Q^{2}\right)+\bar{q}_{i}\left(x, Q^{2}\right)\right]
$$

and likewise for the polarized distribution $\Delta \Sigma$.

The unpolarized and polarized cross sections $\sigma$ and $\Delta \sigma$ are respectively the sum and the difference of the cross sections with fixed parallel and antiparallel proton and gluon helicity. We have included a contribution from the scattering of the incoming gluon off quark and antiquark partons in the proton, which is present at higher orders even though it vanishes at LO where only the diagram of fig. 1a contributes. If we then expand the coefficient functions (i.e. rescaled partonic cross sections) $C^{h}$ and $\Delta C^{h}$ in powers of $\alpha_{s}$ it follows immediately that the LO term in the expansion is given by eq. (2.10). Positivity of the cross sections with fixed gluon and proton helicities then implies

$$
\left|\Delta \sigma\left(x, m_{h}^{2}\right)\right| \leq \sigma\left(x, m_{h}^{2}\right)
$$

and thus the LO bound eq. (2.11). We conclude that the naive partonic positivity bound eqs. (2.8), (2.11) hold for quarks and gluons provided the scale is large enough that NL and higher order corrections are negligible.

Since parton distributions themselves are scale dependent, one is led to ask how the positivity bounds will vary with scale at LO [6]. The scale dependence of parton distributions is expressed in terms of the singlet distribution eq. (2.14) and nonsinglet quark distributions defined as

$$
q_{\mathrm{NS}}\left(x, Q^{2}\right)=\sum_{i=1}^{n_{f}}\left(\frac{e_{i}^{2}}{\left\langle e^{2}\right\rangle}-1\right)\left(q_{i}\left(x, Q^{2}\right)+\bar{q}_{i}\left(x, Q^{2}\right)\right),
$$

and likewise for the polarized distributions $\Delta q_{\mathrm{NS}}$. The moments of the nonsinglet distributions then evolve multiplicatively:

$$
q_{\mathrm{NS}}\left(N, Q^{2}\right)=\left(\frac{\alpha_{s}\left(Q_{0}^{2}\right)}{\alpha_{s}\left(Q^{2}\right)}\right)^{a_{\mathrm{NS}}(N)} q_{\mathrm{NS}}\left(N, Q_{0}^{2}\right)
$$

where for a generic distribution $f(x)$ we have defined $f(N)=\int_{0}^{1} d x x^{N-1} f(x)$. Moments of the singlet quark evolve by mixing with the gluon, according to

$$
\begin{aligned}
& {\left[c_{ \pm}^{1}(N) \Sigma\left(N, Q^{2}\right)+c_{ \pm}^{2}(N) g\left(N, Q^{2}\right)\right]=} \\
& \quad=\left(\frac{\alpha_{s}\left(Q_{0}^{2}\right)}{\alpha_{s}\left(Q^{2}\right)}\right)^{a_{ \pm}(N)}\left[c_{ \pm}^{1}(N) \Sigma\left(N, Q_{0}^{2}\right)+c_{ \pm}^{2}(N) g\left(N, Q_{0}^{2}\right)\right]
\end{aligned}
$$

and likewise for polarized distributions.

Now, the LO anomalous dimensions (which are the only relevant ones asymptotically) satisfy $a_{+}(N) \geq a_{N S}(N) \geq a_{-}(N)$ as it is trivial to verify from their explicit expression [1]. 
It follows that the largest singlet anomalous dimension $a_{+}(N)$ will asymptotically drive the evolution of all the quark distributions for each flavour and of the gluon distribution. The same of course applies to polarized anomalous dimensions $\Delta a(N)$. It is also easy to see that necessarily $a_{+}(N) \geq\left|\Delta a^{+}(N)\right|$ for all $N$ : this is an immediate consequence of the fact that LO unpolarized and polarized anomalous dimensions are respectively sums and differences of the square moduli of invariant particle emission vertices [7] (up to virtual contributions which are the same in the polarized and unpolarized case). Indeed, it is easy to check from their explicit expressions [1] that for all finite $N, a_{+}(N)>\left|\Delta a_{+}(N)\right|$, while $\left.\lim _{N \rightarrow \infty} a_{+}(N)=\lim _{N \rightarrow \infty} \Delta a_{+}(N)\right)$. It follows that for all finite $N$

$$
\lim _{Q^{2} \rightarrow \infty} \frac{\left|\Delta q_{i}\left(N, Q^{2}\right)\right|}{q_{i}\left(N, Q^{2}\right)}=\frac{\left|\Delta g\left(N, Q^{2}\right)\right|}{g\left(N, Q^{2}\right)}=0 .
$$

Hence, the LO bounds eqs. (2.8), (2.11) will always be satisfied at large enough scale. Notice that, conversely, this implies that the LO bounds will always be violated at sufficiently low scale, or, otherwise stated, the (scale-independent) LO bounds become more and more restrictive as the scale is decreased, i.e. they become unacceptably restrictive if imposed at an excessively low scale. On the other hand, this raises the issue of computing subleading corrections to the bounds. We will do this in the next section.

\section{Positivity beyond leading order}

At NLO and beyond helicity-flip cross sections are no longer zero, so that the polarized and unpolarized coefficient functions $C$ and $\Delta C$ are no longer equal to each other. In addition, the gluon DIS coefficient function $C_{g}^{d}$ and the quark Higgs production coefficient function $C_{s}^{h}$ become nonzero. As a consequence, the LO positivity bounds, eqs. (2.8), (2.11) receive NL corrections. In order to discuss these corrections, it is convenient to first rewrite the structure functions eqs. (2.3)-(2.4) by separating the quark singlet and nonsinglet, rather than individual flavours, since the NLO bounds are affected by quark-gluon mixing, but this only involves the singlet quark distribution:

$$
\begin{aligned}
& F_{1}\left(x, Q^{2}\right)=\frac{\left\langle e^{2}\right\rangle}{2}\left[C_{N S}^{d} \otimes q_{N S}+C_{s}^{d} \otimes \Sigma+2 n_{f} C_{g}^{d} \otimes g\right], \\
& g_{1}\left(x, Q^{2}\right)=\frac{\left\langle e^{2}\right\rangle}{2}\left[\Delta C_{N S}^{d} \otimes \Delta q_{N S}+\Delta C_{s}^{d} \otimes \Delta \Sigma+2 n_{f} \Delta C_{g}^{d} \otimes \Delta g\right] .
\end{aligned}
$$

The LO positivity bounds on individual quark and antiquark flavours eq. (2.8) then imply LO positivity of the C-even combination

$$
\left|\Delta q_{i}\left(x, Q^{2}\right)+\Delta \bar{q}_{i}\left(x, Q^{2}\right)\right| \leq\left[q_{i}\left(x, Q^{2}\right)+\bar{q}_{i}\left(x, Q^{2}\right)\right]
$$

which in turn implies LO positivity of the singlet quark distribution, eq. (2.14)

$$
\left|\Delta \Sigma\left(x, Q^{2}\right)\right| \leq \Sigma\left(x, Q^{2}\right)
$$

This is of course a necessary, but not sufficient condition for LO positivity of individual flavours, eq. (3.3), to hold. A necessary and sufficient condition for eq. (3.3) is given 
by constraining the polarized singlet by eq. (3.4), and the polarized nonsinglet through constraints which depend both on the value of $\Delta \Sigma$ and on the unpolarized (singlet and nonsinglet) distributions. The construction of such constraints is trivial: consider for instance the simple two-flavour case. The constraints eq. (3.3) then mean that a rectangular area is allowed in the $(\Delta u, \Delta d)$ plane. The corresponding equivalent constraints on $\Delta \Sigma$ and $\Delta q_{N S}$ are found by giving coordinates in the plane with respect to the $\Delta \Sigma-\Delta q_{N S}$ axes, which are rotated by $\pi / 4$ with respect to the original axes. Likewise, for the separate positivity of quarks and antiquarks (eq. (2.8)): necessary and sufficient conditions can be derived by imposing positivity of the $\mathrm{C}-$-even combination, eq. (3.3), and a similarly derived condition on the $\mathrm{C}-$ odd combination $\Delta q_{i}-\Delta \bar{q}_{i}$.

We are now ready to discuss the NLO modification of the quark and gluon bounds eqs. (2.8),(2.11). First, note that the bounds on physical cross sections eqs. (2.2)-(2.15) must be satisfied for all $x$, and therefore also by the corresponding Mellin moments:

$$
\begin{aligned}
\left|g_{1}\left(N, Q^{2}\right)\right| & \leq F_{1}\left(N, Q^{2}\right) \\
\left|\Delta \sigma\left(N, Q^{2}\right)\right| & \leq \sigma\left(N, Q^{2}\right) .
\end{aligned}
$$

Since the bound eq. (3.5) must hold for any target, the same arguments which lead from eq. (2.7) to eq. (3.4) now give the general bound on the singlet quark distribution

$$
\begin{aligned}
\mid \Delta C_{s}^{d}\left(N, Q^{2}\right) \Delta \Sigma\left(N, Q^{2}\right) & +2 n_{f} \Delta C_{g}^{d}\left(N, Q^{2}\right) \Delta g\left(N, Q^{2}\right) \mid \\
\leq & {\left[C_{s}^{d}\left(N, Q^{2}\right) \Sigma\left(N, Q^{2}\right)+2 n_{f} C_{g}^{d}\left(N, Q^{2}\right) g\left(N, Q^{2}\right)\right] }
\end{aligned}
$$

which at NLO implies

$$
\frac{\left|\Delta \Sigma\left(N, Q^{2}\right)\left(1+\frac{\alpha_{s}}{2 \pi} \Delta C_{s}^{d,(1)}\left(N, Q^{2}\right)\right)+2 n_{f} \frac{\alpha_{s}}{2 \pi} \Delta C_{g}^{d,(1)}\left(N, Q^{2}\right) \Delta g\left(N, Q^{2}\right)\right|}{\Sigma\left(N, Q^{2}\right)\left(1+\frac{\alpha_{s}}{2 \pi} C_{s}^{d,(1)}\left(N, Q^{2}\right)\right)+2 n_{f} \frac{\alpha_{s}}{2 \pi} C_{g}^{d,(1)}\left(N, Q^{2}\right) g\left(N, Q^{2}\right)} \leq 1
$$

Similarly, eq. (3.6) gives the NLO bound on the gluon distribution

$$
\frac{\left|\Delta g\left(N, Q^{2}\right)\left(1+\frac{\alpha_{s}}{2 \pi} \Delta C_{g}^{h,(1)}\left(N, Q^{2}\right)\right)+\frac{\alpha_{s}}{2 \pi} \Delta C_{s}^{h,(1)}\left(N, Q^{2}\right) \Delta \Sigma\left(N, Q^{2}\right)\right|}{g\left(N, Q^{2}\right)\left(1+\frac{\alpha_{s}}{2 \pi} C_{g}^{h,(1)}\left(N, Q^{2}\right)\right)+\frac{\alpha_{s}}{2 \pi} C_{s}^{h,(1)}\left(N, Q^{2}\right) \Sigma\left(N, Q^{2}\right)} \leq 1
$$

Equivalently, we may reabsorb the NL modification of the bounds in a change of factorization scheme. Indeed, we are always free to choose to work in a "parton" scheme in which the coefficient functions for the defining process, computed to any given perturbative order, coincide with their LO expressions

$$
\begin{aligned}
& C_{s}^{d}(N)=C_{g}^{h}(N)=\Delta C_{s}^{d}(N)=\Delta C_{g}^{h}(N)=1 \\
& C_{g}^{d}(N)=C_{s}^{h}(N)=\Delta C_{g}^{d}(N)=\Delta C_{s}^{h}(N)=0 .
\end{aligned}
$$

Imposing that all higher order terms vanish $C^{d,(i)}=C^{h,(i)}=\Delta C^{d,(i)}=\Delta C^{h,(i)}=0$, $i \geq 1$ determines the factorization scheme completely: given coefficient functions up to, 
say, NLO in any scheme (such as the $\overline{\mathrm{MS}}$ scheme) we can transform to the parton scheme by redefining the singlet quark and gluon distributions according to

$$
\left(\begin{array}{c}
\Sigma^{\operatorname{parton}}\left(N, Q^{2}\right) \\
g^{\text {parton }}\left(N, Q^{2}\right)
\end{array}\right)=\left[\mathbb{1}+\frac{\alpha_{s}\left(Q^{2}\right)}{2 \pi}\left(\begin{array}{ll}
C_{s}^{d,(1)}(N) & C_{g}^{d,(1)}(N) \\
C_{s}^{h,(1)}(N) & C_{g}^{h,(1)}(N)
\end{array}\right)\right]\left(\begin{array}{c}
\Sigma\left(N, Q^{2}\right) \\
g\left(N, Q^{2}\right)
\end{array}\right)
$$

and likewise for polarized parton distributions. Otherwise stated, we are always free to choose a scheme in which the bounds reduce to their naive LO form. However, in a generic scheme parton distributions at NLO and beyond will not necessarily respect the LO positivity constraints eqs. (2.8), (2.11). Nevertheless, once we impose the correct positivity bounds eq. (3.8)-(3.9) on NLO parton distributions in any scheme of our choice, physical cross sections will be positive up to NNLO corrections, and if the parton distributions are transformed to any other scheme they will respect the positivity bound pertinent to that scheme.

The coefficient functions $C^{d}, \Delta C^{d}$ and $C^{h}$ are all known up to NLO. In order to determine the full set of NLO positivity bounds and study their phenomenological implications we must thus still determine the NLO polarized quark and gluon coefficient functions $\Delta C^{h}$. We will do this in the next section.

\section{Determination of the polarized Higgs production coefficient functions to next-to-leading order}

At NLO the process $g+p \rightarrow H+X$ can also proceed through gluon-quark fusion, $g+q \rightarrow H+q$ (fig. 1b), so the quark coefficient functions $C_{q}^{h}, \Delta C_{q}^{h}$ are nonzero; furthermore since there is an extra particle in the final state both helicity configurations can now contribute and the polarized and unpolarized coefficient functions are unequal. Similarly, the gluon-gluon fusion contribution can now proceed with emission of an extra gluon, $g+g \rightarrow H+g$ (figs. 1c,d), so that the gluon polarized and unpolarized coefficient functions are likewise unequal. The LO process finally receives virtual corrections which necessarily have the same momentum and spin dependence of the LO, and cancel IR divergences of the real emission contributions. All these contributions take a simple form when calculated in the limit $\frac{m_{t}}{m_{h}} \rightarrow \infty$, in which the quark loop behaves as a pointlike effective interaction [8]. We will determine the polarized cross section at NLO within this approximation, which, regardless of its phenomenological relevance, is adequate for conceptual purposes. The corresponding unpolarized calculations have been performed in ref. [9] for the real emission diagrams while the virtual corrections have been determined in ref. [10].

The polarized parton cross sections which define the coefficient functions eq. (2.13)

are determined by computing helicity differences of squared matrix elements, i.e., for the emission process $g+f \rightarrow H+f$, with $f=q, g$ we have

$$
\Delta M_{f}(s, t, u) \equiv\left|M_{f}^{\uparrow \uparrow}(s, t, u)\right|^{2}-\left|M_{f}^{\uparrow \downarrow}(s, t, u)\right|^{2}
$$

where $s, t$ and $u$ are the Mandelstam invariants of the process and the arrows refer to the helicities of the incoming $g$ and $f$ partons. This difference can in turn be determined by 
contracting the tensor amplitudes with polarized projectors (more precisely, differences of projectors): for the real gluon emission process $g+g \rightarrow H+g$

$$
\Delta M_{g}(s, t, u)=-M_{g}^{\mu \nu \rho} M_{g}^{* \alpha \beta}{ }_{\rho} P_{\mu \alpha}^{g} P_{\nu \beta}^{g},
$$

where the gluon projector is given by

$$
P_{\mu \nu}^{g}=i \epsilon_{\mu \nu \rho \sigma} \frac{n^{\rho} k^{\sigma}}{n \cdot k}
$$

in terms of the momentum $k$ carried by the gluon line on which the projector is applied and an arbitrary lightlike vector $n$ such that $k \cdot n \neq 0$ (for example, the momentum of the other gluon line). For the quark emission process $g+q \rightarrow H+q$ we have

$$
\Delta M_{q}(s, t, u)=\operatorname{Tr}\left[M_{q}^{\mu} P^{q} \not k M_{g}^{\dagger \nu} \not k^{\prime}\right] P_{\mu \nu}^{g},
$$

where the trace runs over fermion indices, $k$ and $k^{\prime}$ are the incoming and outgoing quark momenta, and the quark projector on the incoming line is given by

$$
P_{q}=-\gamma_{5}
$$

We can now compute $\Delta M_{f}$ in dimensional regularization in $d$ dimensions; the calculation closely parallels that of the unpolarized contribution [9-10]. The polarized calculation requires a recipe to handle the $\gamma_{5}$ matrix and the antisymmetric tensor: we will continue them to $d$ dimensions according to the HVBM [11] prescription, since this will give results in the same scheme in which the NLO anomalous dimensions of all parton distributions have been determined [12]. In the gluon case the tensor amplitudes are given in ref. [9], while in the quark case we can readily calculate the amplitude $M_{q}^{\rho}$ from the diagram of fig. 1b. We get

$$
\begin{aligned}
& \Delta M_{g}(s, t, u)=\alpha_{s}^{3} G_{F}^{d} \frac{64}{3 \sqrt{2} \pi} \frac{m_{h}^{8}+s^{4}-t^{4}-u^{4}}{s t u} \\
& \Delta M_{q}(s, t, u)=\alpha_{s}^{3} G_{F}^{d} \frac{64}{3 \sqrt{2} \pi} \frac{s^{2}-u^{2}}{2 t}
\end{aligned}
$$

where $G_{F}^{d}$ is the $d$-dimensional continuation of the strength of the effective $g g H$ interaction [10]. The form of this continuation is immaterial because it appears as an overall factor in all the separately divergent contributions which add up to the final finite NLO result.

The kinematics for the inclusive process can be entirely specified in terms of $m_{h}^{2}$, $s=\frac{m_{h}^{2}}{x}$, and the center-of-mass scattering angle of the emitted parton $\cos \theta=1-2 y$, in terms of which the remaining Mandelstam invariants are given by

$$
\begin{aligned}
& t=-\left(s-m_{h}^{2}\right)(1-y)=-m_{h}^{2}(1-y) \frac{1-x}{x} \\
& u=-\left(s-m_{h}^{2}\right) y=-m_{h}^{2} y \frac{1-x}{x} .
\end{aligned}
$$


The physical cross-section is obtained by integrating the real emission amplitude over phase space, adding vitual corrections and collinear and ultraviolet counterterms, and supplying the appropriate flux factor:

$$
\Delta \sigma(g+f \rightarrow H+f)=\frac{1}{2 s} \int d \phi_{2} \Delta M_{f}+\Delta \sigma_{f}^{\mathrm{virt}}+\Delta \sigma_{f}^{\mathrm{coll}}+\Delta \sigma_{f}^{u v}
$$

The two-body phase space in $d=2(2-\epsilon)$ dimensions is given by

$$
d \phi_{2}=\frac{1}{8 \pi} \frac{1}{\Gamma(1-\epsilon)}\left(\frac{4 \pi}{s}\right)^{\epsilon}\left(1-\frac{m_{h}^{2}}{s}\right)^{1-2 \epsilon} y^{-\epsilon}(1-y)^{-\epsilon} d y .
$$

The collinear counterterms remove the singularities which correspond to collinear radiation of the final-state parton by the incoming one, and are thus given by

$$
\Delta \sigma_{f}^{\text {coll }}=\int_{x}^{1} \frac{d y}{y} \Delta \sigma_{0}\left(m_{h}^{2}, y\right) \frac{1}{\epsilon} \Gamma(1+\epsilon)(4 \pi)^{\epsilon} P_{g f}\left(\frac{x}{y}\right)
$$

where $P_{g f}$ is the LO QCD splitting function [7], the factor of $\Gamma(1+\epsilon)(4 \pi)^{\epsilon}$ accompanying the $\epsilon$ pole characterizes the $\overline{\mathrm{MS}}$ subtraction scheme, and $\Delta \sigma_{0}\left(m_{h}^{2}, x=m_{h}^{2} / s\right)$ is the polarized Born cross section in $d$ dimensions, which turns out to coincide with the unpolarized four-dimensional cross-section eq. (2.12) up to the replacement $G_{F} \rightarrow G_{F}^{d}$.1. Virtual corrections, as well as the ultraviolet counterterm related to charge renormalization, only contribute to the gluon process, since the quark process only starts at NLO, and are proportional to the Born cross section in $d$ dimensions. They were computed in ref. [10] from which they can be simply obtained by replacing the $d$ dimensional unpolarized Born cross section with its polarized counterpart, and are thus given by

$$
\begin{array}{r}
\Delta \sigma_{g}^{\mathrm{virt}}=\Gamma(1+\epsilon)(4 \pi)^{\epsilon} \Delta \sigma_{0}\left(m_{h}^{2}, x\right)\left(\frac{3}{\epsilon^{2}}-\frac{11}{2}-2 \pi^{2}\right) \\
\Delta \sigma_{g}^{\mathrm{uv}}=\Delta \sigma_{0}\left(m_{h}^{2}, x\right) \frac{11-2 / 3 n_{f}}{4 \pi} \frac{1}{2 \epsilon} \Gamma(1+\epsilon)(4 \pi)^{\epsilon} .
\end{array}
$$

Performing the phase-space integral eq. (4.10) of the square amplitudes eq. (4.7)-(4.6) and adding the collinear counterterms (4.11) and, in the gluon case, the virtual corrections eq. (4.12) we get the physical cross sections for the NLO processes $g+g \rightarrow H+g$ and $g+q \rightarrow H+q$. Double poles in $\epsilon$ cancel between real and virtual contributions to the gluon process. Poles in $\epsilon$ proportional to $\delta(1-x)$ are generated in the real contribution to the gluon process because of the presence of a term proportional to $(1-x)^{-(1+2 \epsilon)}$ which has an IR singularity in the $\epsilon \rightarrow 0$ limit:

$$
\frac{1}{(1-x)^{1+2 \epsilon}}=-\frac{1}{2 \epsilon} \delta(1-x)+\frac{1}{(1-x)_{+}}-2 \epsilon\left(\frac{\ln (1-x)}{1-x}\right)_{+}+\mathcal{O}\left(\epsilon^{2}\right) .
$$

1 Notice that the fact that the dependence on $\epsilon$ of $\Delta \sigma_{0}$ only comes through $G_{F}^{d}$ is nontrivial, and peculiar of the polarized calculation where it follows from the adoption of the HVMB prescription for the antysimmetric tensor. 
These poles cancel against those in the virtual correction eq. (4.12). The collinear simple poles are cancelled by the splitting function counterterms in both the quark and gluon emission diagrams.

The final finite result for the physical cross sections gives us immediately the coefficient functions $\Delta C^{h}$ eq. (2.13) by dividing out the physical Born cross section eq. (2.12):

$$
\begin{aligned}
& \Delta C_{g}^{h,(1)}(x)= 11 \frac{(1-x)^{3}}{x}+\left(11+2 \pi^{2}\right) \delta(1-x) \\
&+2\left[\frac{\ln (1-x)^{2}}{1-x}\right]_{+} \Delta R_{g g}(x)-\ln x \Delta P_{g g}(x) \\
&+2 \ln \left(\frac{m_{h}^{2}}{\mu^{2}}\right) \Delta P_{g g}(x) \\
& \Delta C_{s}^{h,(1)}(x)=2 \frac{(1-x)^{2}}{x}+\Delta P_{g q}(x)\left[\ln \frac{(1-x)^{2}}{x}+\ln \left(\frac{m_{h}^{2}}{\mu^{2}}\right)\right],
\end{aligned}
$$

where $\Delta P_{f g}$ are LO QCD polarized splitting functions,

$$
\frac{\Delta R_{g g}(x)}{(1-x)_{+}}=\left[\Delta P_{g g}(x)-\frac{\beta_{0}}{2} \delta(1-x)\right],
$$

$\beta_{0}=11-\frac{2}{3} n_{f}$ is the leading coefficient of the QCD beta function, and $\mu^{2}$ is a factorization scale. We can now derive the full set of positivity bounds for the quark and gluon distribution. The result eq. (4.14)-(4.15) is of course also in principle interesting for its own sake as a determination of the cross section for Higgs production in polarized gluon-proton scattering.

\section{Phenomenology of next-to-leading order positivity bounds}

We can now collect all results for the eight coefficient functions which determine the NLO positivity bounds for quarks and gluons, eqs. (3.8)-(3.9), in moment space. The $F_{1}$ coefficient functions $\overline{\mathrm{MS}}$ can be determined from the known $F_{2}$ [13] and $F_{L}$ [四] coefficient functions using $F_{1}=\left(F_{2}-F_{L}\right) /(2 x)$. The $g_{1}$ coefficient functions are available [14 in the $\overline{\mathrm{MS}}$ scheme with HVBM prescription; from these it is straightforward to determine them in the $\mathrm{AB}$ version of the $\overline{\mathrm{MS}}$ scheme [15] which allows a comparison to recent determinations of parton distributions [2]. 3 The full Higgs production unpolarized coefficient functions

2 Note that eqs. (4.14)-(4.15) give the coefficient of $\frac{\alpha_{s}}{2 \pi}$ in the perturbative expansion of the coefficient function. The coefficient functions eqs. (4.14)-(4.15) are thus related to the $O\left(\alpha_{s}^{3}\right)$ contributions to the cross section for the process $g+f \rightarrow H+X$ by $\Delta \sigma(g+f \rightarrow H+X)=$ $\frac{\alpha_{s}^{3} G_{F}}{576 \sqrt{2} \pi^{2}} x C_{f}^{h,(1)}(x)$.

3 The $\mathrm{AB}$ scheme is a variant of the $\overline{\mathrm{MS}}-\mathrm{HVBM}$ scheme in polarized DIS which adopts a different subtraction in the definition of the polarized gluon coefficient function. The scheme is constructed in terms of the $\overline{\mathrm{MS}}$ scheme in such a way that only the $z_{q g}$ entry of the scheme change matrix is nonzero. This means that only the $\Delta C_{g}^{d}$ coefficient function is affected by the scheme change, while all other coefficient fucntions are the same as in the $\overline{\mathrm{MS}}$ scheme. 

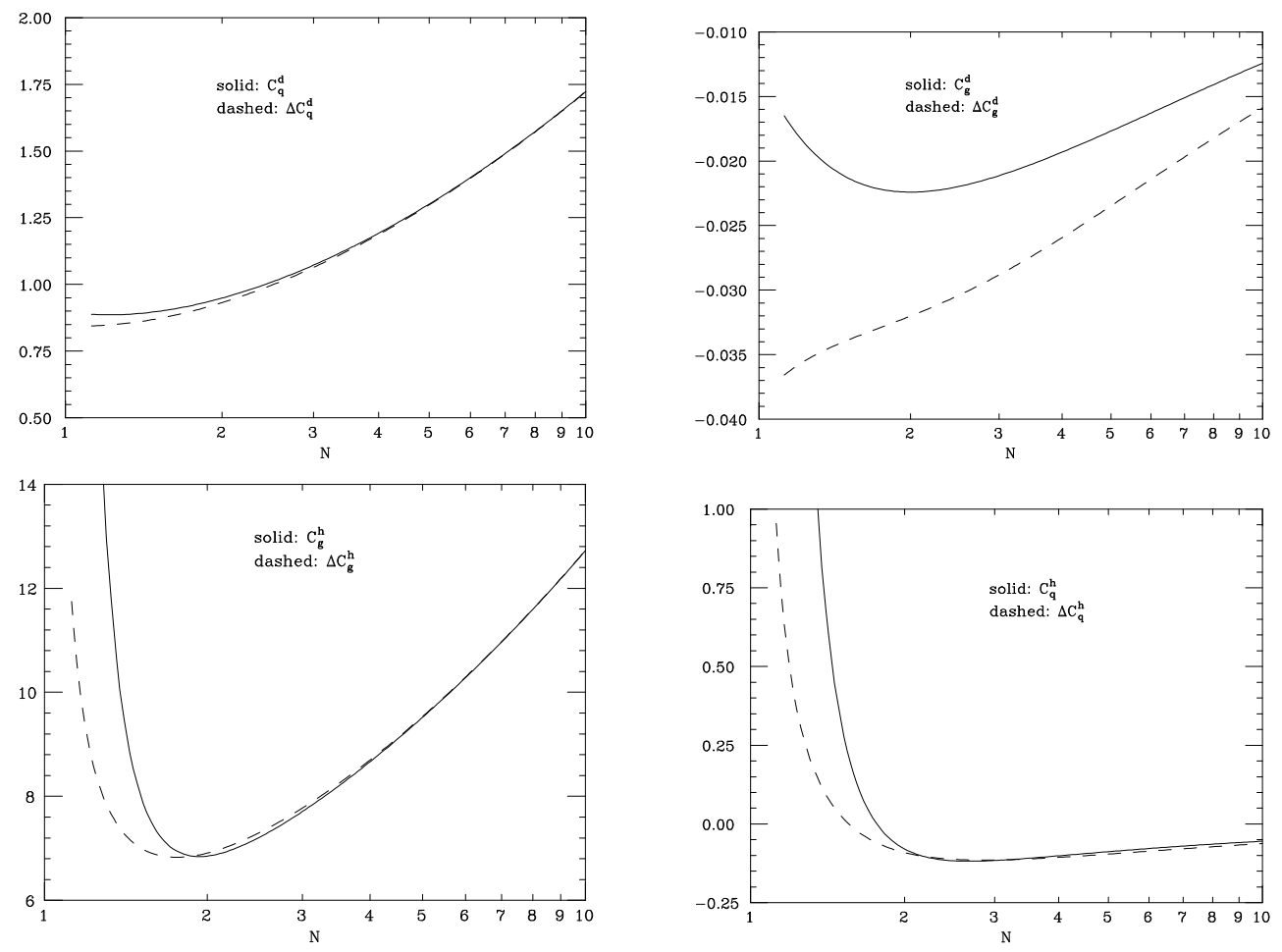

Figure 2: The eight NLO coefficient functions, evaluated at $Q^{2}=1 \mathrm{GeV}^{2}$, as functions of the moment variable $N$.

are given in ref. [10], while the calculation of their polarized counterparts was presented in the previous section. For completeness, we list in Appendix A the full set of NLO polarized and unpolarized coefficient functions in moment space in the $\overline{\mathrm{MS}}-\mathrm{AB}$ scheme.

Combined bounds on $\Delta \Sigma\left(N, Q^{2}\right)$ and $\Delta g\left(N, Q^{2}\right)$ can now be derived using eqs. (3.8)(3.9) for various scales and values of the moment variable. We will assume everywhere the equality of the factorization and renormalization scales; specifically in eq. (4.14), (4.15) $\mu^{2}=m_{h}^{2}$. The bounds are only significant for $N>1$, because the first moments of unpolarized distributions diverge. Furthermore, the scale has to be chosen large enough that the NL truncation of perturbation theory is a reasonable approximation. On the other hand, the bounds will only be relevant at not too large values of the scale, since at asymptotically large scales all bounds are trivially satisfied because of the asymptotic vanishing, eq. (2.19), of the ratios $\frac{\Delta \Sigma}{\Sigma}$ and $\frac{\Delta g}{g}$. Furthermore, at asymptotically large $Q^{2}$, NLO corrections are negligible and all bounds reduce to the naive LO ones discussed in Sect. 2.

In fig. 2 the eight NLO coefficient functions are plotted for $Q^{2}=1 \mathrm{GeV}^{2}$ as functions of the moment variable. It is interesting to note that the NLO corrections to the Higgs production process in the gluon channel (i.e. to $\Delta C_{g}^{h}$ and $C_{g}^{h}$ ) are very large (as large as $100 \%$ at the Higgs scale, and much larger at the low scale considered here). As pointed out in ref. [10], this is mostly due to the very large size of virtual corrections. These corrections, however, are proportional to the Born process and thus only contribute to the gluon channel spin-nonflip cross section, and indeed, $\left|\Delta C_{g}^{h}-C_{g}^{h}\right| \ll\left|\Delta C_{g}^{h}\right|+\left|C_{g}^{h}\right|$. Since the NLO corrections to the positivity bounds are only due to the nonvanishing of 
the spin-flip process, it follows that the NLO corrections to the bounds are actually very small and can be safely treated within perturbation theory despite the large size of the NLO corrections to the coefficient functions themselves.

The pattern of deviations from the LO behavior displayed by the NLO corrections to the coefficient functions of fig. 2 is rather complicated: all the Higgs production coefficient functions satisfy $\left|\Delta C^{h}\right|<C^{h}$ for small $N$ but $\left|\Delta C^{h}\right|>C^{h}$ when $N$ is large; the DIS coefficient functions for all $N$ have $\left|\Delta C_{s}^{d}\right|<C_{s}^{d}$ but $\left|\Delta C_{g}^{d}\right|>C_{g}^{d}$ (notice that both $\Delta C_{g}$ and $C_{g}$ are negative). The fact that some NLO corrections to the polarized coefficient functions are larger than their unpolarized counterparts means that some of the NLO contributions to the associate cross sections are negative.

The bounds eqs. (3.8)-(3.9) are useful to constrain more poorly known parton distributions by the better known ones. Specifically, the bounds are significant for high values of the moment variable $N$ because this singles out the large $x$ region, where the unpolarized distribution is reasonably constrained by the data whereas very little is known about the polarized distribution.

In fig. 3 we present the LO and NLO bounds eqs. (3.8)-(3.9) for several relevant values of the moment variable $N$. The bounds are given in the form of an allowed region in the $\Delta \Sigma-\Delta g$ plane. The contours depend on the unpolarized distributions, which are taken from the CTEQ4LQ set [16]. The maximal allowed values of the unpolarized distributions are thus affected by the uncertainity on the unpolarized distributions. The size of the uncertainty of parton distributions cannot be evaluated precisely at present. However, in the central $x$ region $(0.01 \lesssim x \lesssim 0.8)$ the uncertainty can be considered to be essentially negligible on the unpolarized quark distribution, while a recent analysis [17] suggests that the uncertainty on the unpolarized gluon distribution is of order $10 \%$ unless $x$ is very small $\left(x \lesssim 10^{-3}\right)$ or large $(x \gtrsim 0.2)$ the gluon being vanishingly small at large $x$. This should give an idea on the expected uncertainty on the bounds of fig. 3 due to the errors on parton distributions, while the difference between LO and NLO bounds gives an idea of the uncertainty related to higher order corrections.

The bounds are presented at the scale $Q^{2}=1 \mathrm{GeV}^{2}$ which is often chosen as "initial" scale in parametrization of parton distributions, and which can be taken as a lower limit for the applicability of perturbation theory. The NLO corrections are seen to modify and distort the allowed area in the $\Delta \Sigma-\Delta g$ plane. The pattern of modification of the LO bounds follows the pattern of NLO corrections to the coefficient functions displayed in fig. 2. In particular, it is clear that if $\left|\Delta C^{(1)}(N)\right| \leq C^{(1)}(N)$ (i.e. the NLO contributions to the various cross sections are all positive) then the NLO bound is less restrictive than the LO bound. For instance the quark bound becomes

$$
\begin{gathered}
-\frac{\left[C_{s}^{d}\left(N, Q^{2}\right) \Sigma\left(N, Q^{2}\right)+2 n_{f}\left(C_{g}^{d}\left(N, Q^{2}\right) g\left(N, Q^{2}\right)+\Delta C_{g}^{d}\left(N, Q^{2}\right) \Delta g\left(N, Q^{2}\right)\right)\right]}{\Delta C_{s}^{d}\left(N, Q^{2}\right)} \\
\leq \Delta \Sigma\left(N, Q^{2}\right) \leq \\
\frac{\left[C_{s}^{d}\left(N, Q^{2}\right) \Sigma\left(N, Q^{2}\right)+2 n_{f}\left(C_{g}^{d}\left(N, Q^{2}\right) g\left(N, Q^{2}\right)-\Delta C_{g}^{d}\left(N, Q^{2}\right) \Delta g\left(N, Q^{2}\right)\right)\right]}{\Delta C_{s}^{d}\left(N, Q^{2}\right)} .
\end{gathered}
$$



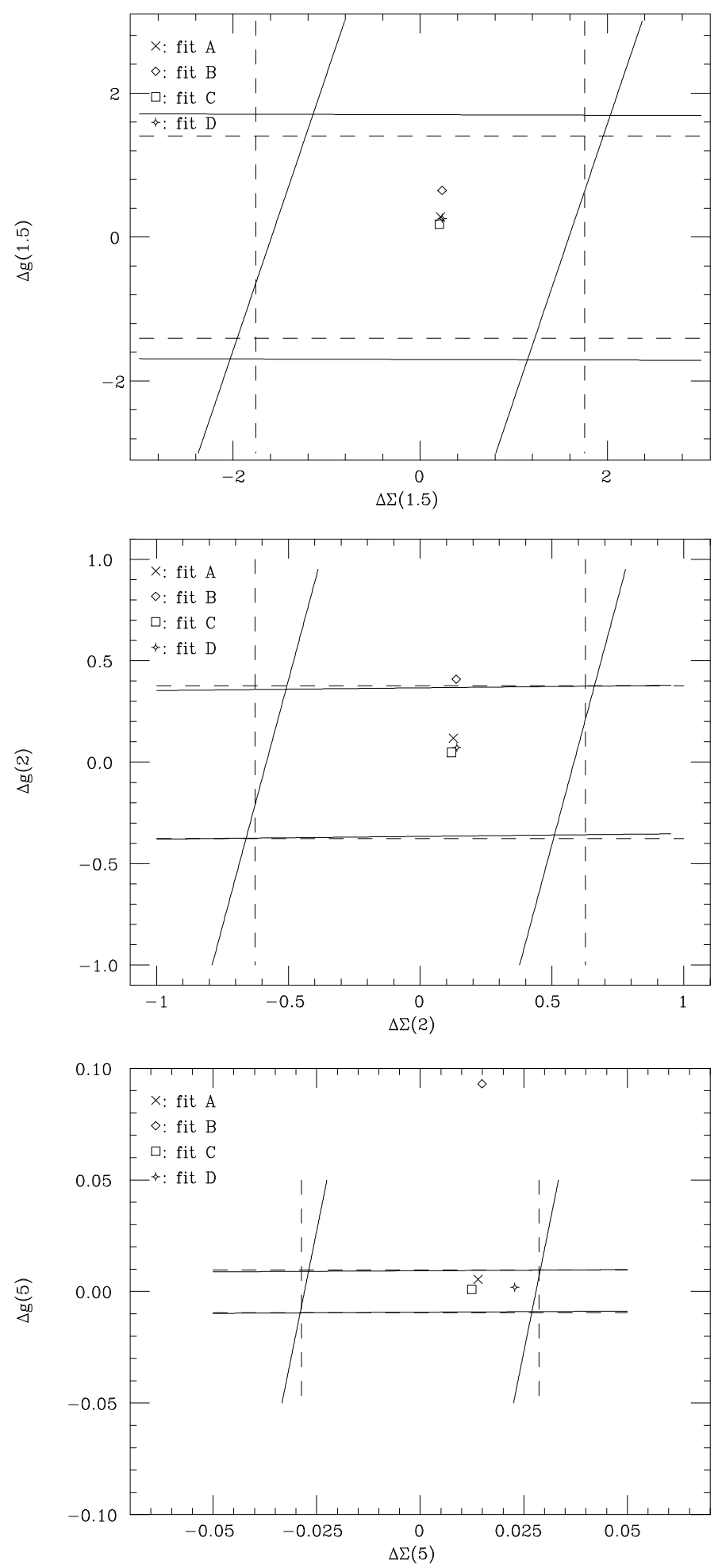

Figure 3: The LO (dashed lines) and NLO (solid lines) positivity bounds on $\Delta \Sigma(N)$ and $\Delta g(N)$ for $Q^{2}=1 \mathrm{GeV}^{2}$ and $N=1.5,2,5$. The values of $\Delta \Sigma$ and $\Delta g$ corresponding to the NLO fits to $g_{1}$ data of ref. [2] are also shown. 
This is always less restrictive than

$$
\left|\Delta \Sigma\left(N, Q^{2}\right)\right| \leq \frac{C_{s}^{d}\left(N, Q^{2}\right)}{\Delta C_{s}^{d}\left(N, Q^{2}\right)} \Sigma\left(N, Q^{2}\right)
$$

because $C_{g}^{d}\left(N, Q^{2}\right) g\left(N, Q^{2}\right) \pm \Delta C_{g}^{d}\left(N, Q^{2}\right) \Delta g\left(N, Q^{2}\right)$ is always positive if $\left|\Delta C_{g}^{d}\right| \leq C_{g}^{d}$ since to NLO accuracy we can use eq. (2.11) for the gluon contribution. Eq. (5.2) is manifestly less restrictive than the LO bound eq. (3.4) if $\left|\Delta C_{s}^{d}\right| \leq C_{s}^{d}$. If instead $\left|\Delta C_{s}^{d}\right|>C_{s}^{d}$ the NLO bound will be more restrictive if the gluon contribution can be neglected (so that eq. (5.2) holds) but could still be more restrictive than the LO one if the gluon contribution is large. Similar arguments apply to the gluon distribution.

Inspection of fig. 3 shows that at $Q^{2}=1 \mathrm{GeV}^{2}$ the NLO corrections to the LO bounds turn out to be reasonably small, but non-negligible for small $N$-moments, where they tend to make the bound less restrictive than the LO bound if both $\Delta \Sigma$ and $\Delta g$ are positive (as experiments appear to indicate). The corrections are instead essentially negligible for large values of $N$. However, at sufficiently low scale all the NLO corrections will start growing and dramatically modify the LO bounds, but use of perturbation theory at such scales is not advisable.

Figure 3 also shows the values of $\Delta \Sigma$ and $\Delta g$ obtained in a recent determination [2] of polarized parton distributions. Different fits correspond to different choices of functional forms and theoretical assumptions on the parton distributions, and give a feeling for the spread of the results. It is apparent that one fits (fit B) display large percentage violations of the positivity bound on $\Delta g(N)$ for large values of the moment variable $N$. This reflects the fact that large values of $N$ correspond to the large $x$ region where $\Delta g(N)$ is extremely small and the fitted results are affected by very large uncertainties. Indeed, the main purpose of ref. [2] was instead to provide a reliable determination of the first moments of $\Delta \Sigma$ and $\Delta g$, while the large $x$ i.e. large $N$ behavior of the parton distributions could not be determined accurately. This however shows explicitly how positivity bounds can be used to improve a determination of parton distributions, and specifically $\Delta g$ by providing independent information. Indeed, we could modify fit B in such a way that the bounds are respected, without significantly affecting the quality of the fit to data and the determination of the first moment. However, imposing that the bound is satisfied we would get a fit with reduced uncertainity on $\Delta g$ in the large $x$ region.

\section{Summary and outlook}

In the naive parton model the polarized parton densities in a proton with helicity $+1 / 2$, $p_{+}$and $p_{-}$, are interpreted as probability densities and, as a consequence, are positive semidefinite. Thus, given $\Delta p=p_{+}-p_{-}$and $p=p_{+}+p_{-}$, they satisfy $|\Delta p| \leq p$, for any $p=q_{i}, \bar{q}_{i}, g$. The QCD-improved parton model reproduces the naive parton results at LO, i.e. when all higher order perturbative corrections (as well as all power corrections) are neglected. In this limit, if the naive positivity bounds for parton densities were violated, one could exhibit hard processes with unacceptable negative cross-sections. For quarks obvious examples of such processes are given by the polarized asymmetries $A_{1}=g_{1} / F_{1}$ eq. (2.1). For gluons, the selection of the process which leads to the bound $|\Delta g| \leq g$ is less 
direct. One needs a process whose rate on a polarized proton with helicity $+1 / 2$ starts with $g_{+}$and does not contain either $g_{-}$or $q_{ \pm}$or $\bar{q}_{ \pm}$. The process $g+p \rightarrow H+X$, where $H$ is a neutral, colourless massive scalar (for example, a Higgs boson) satisfies the required properties.

As is well known, most naive parton model properties and sum rules are in general violated if higher order QCD corrections are included. For example, the charge sum rules $u\left(1, Q^{2}\right)-\bar{u}\left(1, Q^{2}\right)=2$ and similar ones [where $u\left(N, Q^{2}\right)$ is the N-th moment of the $\mathrm{u}-$ quark density $u\left(x, Q^{2}\right)$ ] are in general violated, unless the unpolarized quark densities are defined by the structure function $F_{2}$, 18] i.e. by extending to all orders, by definition, the LO expression of $F_{2}$ in terms of quark densities. Similarly also the momentum sum rule or the LO positivity conditions are in general violated at NLO and higher accuracy, with corrections which depend on the definitions of the relevant quantities beyond LO.

In this article we have computed the NLO corrections to the positivity relations in a set of specified definitions. In particular we adopted the $\overline{\mathrm{MS}}$ scheme and a simple set of physical processes for the definition of parton densities, including the above mentioned Higgs production process for the gluon definition. Of particular interest in practice are the positivity bounds in the polarized singlet sector $\Delta \Sigma, \Delta g$. This is because the determination of the polarized gluon density attracts considerable attention at present. When fitting the data on scaling violations often the gluon comes close to the LO positivity bound at large $x$, so that the precise form of the bound at NLO can be relevant. While at LO the positivity bounds in the singlet sector are given separately for $\Delta \Sigma(N)$ and $\Delta g(N)(|\Delta \Sigma(N)| \leq \Sigma(N)$, $|\Delta g(N)| \leq g(N))$, at NLO due to quark-gluon mixing the allowed domain is modified into a more complicated region of the $\Delta \Sigma-\Delta g$ plane.

Our detailed quantitative results show that the modified region in the $\Delta \Sigma-\Delta g$ plane can be either more or less restrictive than the LO positivity domain, depending on the values of $\mathrm{N}, \Sigma(N)$ and $g(N)$. Interestingly, we also find that the corrections to the positivity limiting contours are in general much smaller than the size of the corrections on the individual coefficient functions. In particular this is true for the corrections to the Higgs process. It is well known that the NLO corrections to Higgs production by gluon-gluon fusion are quite large [10]. But the bulk of the corrective terms is from virtual diagrams which, being proportional to the Born term, do not modify the LO bounds.

As a consequence, we find that, even at relatively small values of $Q^{2}$, such as $Q^{2} \sim 1 \mathrm{GeV}^{2}$, the modified domains are reasonably close to the corresponding LO region. Of course if $Q^{2}$ is further lowered down to a clearly non-perturbative region, the NLO corrections eventually explode and the NLO positivity contours become very different than the LO contours. It is important to note that if the LO positivity bounds are imposed at very small $Q^{2}$ they in general produce exaggerately constraining limits on the parton densities. This is because the evolution goes in the direction of making the positivity bounds trivial at $Q^{2} \rightarrow \infty$. Hence, applying the LO bounds at very small $Q^{2}$ leads to an evolved domain at larger $Q^{2}$ that is considerably smaller than the LO bound at the larger value of $Q^{2}$. It follows that if parton distributions are parametrized at a low scale, positivity bounds can only be safely imposed at a higher scale, where perturbation theory is reliable. A possible strategy is to use positivity bounds to discard fitted parton distributions which do not satisfy them, thereby reducing the uncertainty on the results of the fit. 
Imposing positivity bounds consistently at NLO in a given scheme will then guarantee positivity of physical cross sections for the defining processes. It will also guarantee positivity of any other process whose LO coefficient functions do not have the partonic form eqs. (2.6), (2.10), because the LO bounds for such processes will be less restrictive. It will not, however, guarantee positivity of other processes for which eqs. (2.6), (2.10) hold, because then positivity would depend on the relative sign and size of NLO corrections.

In conclusion, positivity bounds on parton distributions derived from specific defining processes are in general useful as necessary conditions which provide complementary information on polarized parton distributions, especially at large $x$.

Acknowledgements: We thank M. Anselmino, R. D. Ball and M. Spira for discussions and correspondence. This work was supported in part by the EU Fourth Framework Programme "Training and Mobility of Researchers", Network 'Quantum Chromodynamics and the Deep Structure of Elementary Particles', contract FMRX-CT98-0194 (DG 12-MIHT). 


\section{References}

[1] See e.g. G. Altarelli, Phys. Rep. 81 (1982) 1.

[2] G. Altarelli, R. D. Ball, S. Forte and G. Ridolfi, Nucl. Phys. B496 (1997) 337; hep-ph/9803237.

[3] See e.g. M. Anselmino, A. Efremov and E. Leader, Phys. Rep. 261 (1995) 1.

4 A. Zee, F. Wilczek and S. B. Treiman, Phys. Rev. D10 (1974) 2881.

5. F. Wilczek, Phys. Rev. Lett. 39 (1977) 1304.

6. C. Bourrely, E. Leader and O. V. Teryaev, hep-ph/9803238.

7. G. Altarelli and G. Parisi, Nucl. Phys. B126 (1977) 298.

8 A. Vainshtein, V. Zakharov and M. Shifman, Sov. Phys. Usp. 23 (1980) 429.

[9] R. K. Ellis et al., Nucl. Phys. B297 (1988) 221.

[10] S. Dawson, Nucl. Phys B359 (1991) 283;

A. Djouadi, M. Spira and P. M. Zerwas, Phys. Lett. B264 (1991) 440.

[11] G. 't Hooft and M. Veltman, Nucl. Phys B44 (1972) 189;

P. Breitenlohner and D. Maison, Comm. Math. Phys. 52 (1977) 11.

[12] R. Mertig and W. L. van Neerven, Zeit. Phys. C70 (1996) 637;

W. Vogelsang, Phys. Rev. D54 (1996) 2023, Nucl. Phys. B475 (1996) 47.

[13] W. A. Bardeen et al, Phys. Rev. D18 (1978) 3998.

14. Jodaira, Nucl. Phys. B165 (1980) 129.

15. R.D. Ball, S. Forte and G. Ridolfi, Phys. Lett. B378 (1996) 255.

16. H. L. Lai et al., Phys. Rev. D55 (1997) 1280.

17 H. L. Lai et al., hep-ph/9801444.

[18] G. Altarelli, R. K. Ellis and G. Martinelli, Nucl. Phys. B143 (1978) 521; B157 (1979) 461. 


\section{Appendix A. Coefficient functions in moment space}

We list here the Mellin moments $C(N) \equiv \int_{0}^{1} x^{N-1} C(x) d x$ of the NLO contributions to the coefficient functions in the $\overline{\mathrm{MS}}$ scheme for generic values of the ratio of factorization and renormalization scales $k \equiv \frac{\mu_{R}^{2}}{\mu_{F}^{2}}$. The full coefficient functions are defined in eq. (2.5); the LO contributions are given in eqs. (2.6), (2.10).

Deep-inelastic scattering NLO coefficient functions:

$$
\begin{aligned}
& C_{S}^{d,(1)}(N)=C_{F}\left[S_{1}(N-1)\left(S_{1}(N+1)+\frac{3}{2}\right)+\frac{4}{N}-\frac{1}{N+1}-\frac{9}{2}\right. \\
& \left.-S_{2}(N-1)\right]+P_{q q} \ln k \\
& C_{g}^{d,(1)}(N)=-T_{R}\left[S_{1}(N-1) \frac{N^{2}+N+2}{N(N+1)(N+2)}+\frac{1}{N+2}\right] \\
& +P_{q g} \ln k \\
& \Delta C_{S}^{d,(1)}(N)=C_{F}\left[S_{1}(N-1)\left(\frac{3}{2}+S_{1}(N+1)\right)\right. \\
& \left.-S_{2}(N-1)-\frac{9}{2}+\frac{3}{N}\right]+\Delta P_{q q}(N) \ln k \\
& \Delta C_{g}^{d,(1)}(N)=-T_{R} \frac{N-1}{N(N+1)}\left[S_{1}(N-1)+1\right]+\Delta P_{q g}(N) \ln k .
\end{aligned}
$$

The AB- $\overline{\mathrm{MS}}$ scheme [15] (used in sect. 5) is obtained adding to $\Delta C_{S}^{d,(1)}(N)$ eq. (A.4) a contribution equal to $\frac{-T_{R}}{N}$, while all other coefficient functions are left unchanged.

Higgs production NLO coefficient functions:

$$
\begin{gathered}
C_{g}^{h,(1)}(N)=C_{A}\left[\frac{11+4 \pi^{2}}{3}-\frac{22}{N\left(N^{2}-1\right)(N+2)}\right] \\
+2 Q_{g g}(N)+2 P_{g g}(N) \ln k \\
C_{S}^{h,(1)}(N)=C_{F} \frac{N^{2}-N-3}{N\left(N^{2}-1\right)}+Q_{g q}(N)+P_{g q}(N) \ln k \\
\Delta C_{g}^{h,(1)}(N)=C_{A}\left[\frac{11+4 \pi^{2}}{3}+\frac{22}{N\left(N^{2}-1\right)(N+2)}\right] \\
\Delta C_{S}^{h,(1)}(N)=C_{F} \frac{3}{N\left(N^{2}-1\right)}+\Delta Q_{g g}(N)+2 \Delta P_{g g}(N) \ln k
\end{gathered}
$$

We have defined

$$
S_{i}(N)=\sum_{k=1}^{N} \frac{1}{k^{i}}
$$




$$
\begin{gathered}
Q_{g g}(N)=2 C_{A}\left[S_{1}^{2}(N+2)-2 S_{1}^{2}(N+1)\right. \\
\left.+3 S_{1}^{2}(N)-2 S_{1}^{2}(N-1)+S_{1}^{2}(N-2)\right] \\
\Delta Q_{g g}(N)=2 C_{A}\left[2 S_{1}^{2}(N+1)-3 S_{1}^{2}(N)+2 S_{1}^{2}(N-1)\right] \\
Q_{g q}(N)=C_{F}\left[-\frac{4 S_{1}(N-1)}{N-1}+\frac{4 S_{1}(N)}{N}-\frac{2 S_{1}(N+1)}{N+1}\right. \\
\left.+\frac{2}{(N-1)^{2}}-\frac{2}{N^{2}}+\frac{1}{(N+1)^{2}}\right] \\
\Delta Q_{g q}(N)=C_{F}\left[-\frac{4 S_{1}(N)}{N}+\frac{2 S_{1}(N+1)}{N+1}+\frac{2}{N^{2}}-\frac{1}{(N+1)^{2}}\right]
\end{gathered}
$$

Furthermore, $P_{i j}(N)$ and $\Delta P_{i j}(N)$ are the usual LO polarized and unpolarized anomalous dimensions, i.e. the Mellin transforms of the LO QCD splitting functions, whose expressions we list here for completeness:

$$
\begin{aligned}
P_{q q} & =C_{F}\left[\frac{3}{2}-\frac{1}{N}-\frac{1}{N+1}-2 S_{1}(N-1)\right] \\
P_{q g} & =T_{R} \frac{N^{2}+N+2}{N(N+1)(N+2)} \\
P_{g q} & =C_{F} \frac{N^{2}+N+2}{N\left(N^{2}-1\right)} \\
P_{g g} & =2 C_{A}\left[\frac{1}{N(N-1)}+\frac{1}{(N+1)(+2)}-S_{1}(N)\right]+\frac{\beta_{0}}{2} \\
\Delta P_{q q} & =C_{F}\left[\frac{3}{2}-\frac{1}{N}-\frac{1}{N+1}-2 S_{1}(N-1)\right] \\
\Delta P_{q g} & =T_{R} \frac{N-1}{N(N+1)} \\
\Delta P_{g q} & =C_{F} \frac{N+2}{N(N+1)} \\
\Delta P_{g g} & =2 C_{A}\left[\frac{2}{N(N+1)}-S_{1}(N)\right]+\frac{\beta_{0}}{2} .
\end{aligned}
$$

